\title{
Significance of Urinary Liver-fatty Acid-binding Protein in Cardiac Catheterization in Patients with Coronary Artery Disease
}

\author{
Yusuke Fukuda, Shin-ichiro Miura, Bo Zhang, Atsushi Iwata, Akira Kawamura, \\ Hiroaki Nishikawa, Kazuyuki Shirai and Keijiro Saku
}

\begin{abstract}
Objectives We investigated the significance of urinary liver fatty acid-binding protein (U-L-FABP) monitoring during cardiac catheterization in patients with cardiovascular disease (CVD).

Methods The subjects included 27 consecutive patients with stable angina (SAP group) or acute coronary syndrome (ACS group) who had undergone successful percutaneous coronary intervention (PCI), and 12 patients were also enrolled as controls (C group). Urinary and serum parameters were measured immediately before and after and 1 day after PCI.

Results The ratio of U-L-FABP to U-creatinine (U-Cr) (U-L-FABP/U-Cr) in the ACS group was significantly higher than those in both the SAP and C groups before PCI. In addition, none of the patients in the SAP group showed contrast-induced nephropathy (CIN) based on the levels of serum (S)-Cr and U-L-FABP/ $\mathrm{U}-\mathrm{Cr}$ after PCI. Although none of the patients in the ACS group showed CIN according to S-Cr, the level of U-L-FABP/U-Cr was continuously high throughout the study period. Moreover, since there were significant differences in U-L-FABP/U-Cr, U-N-acetyl- $\beta$-D-glucosaminidase, S-uric acid and \% medication with calcium channel blockers before PCI between the ACS and SAP groups, a multiple regression analysis was performed using these parameters. It showed that U-L-FABP/U-Cr was most closely associated with the classification of SAP and ACS $(\mathrm{p}<0.0001)$. The cut-off level for the greatest sensitivity and specificity for U-L-FABP for the diagnosis of ACS was $13.4 \mu \mathrm{g} / \mathrm{g} \cdot \mathrm{Cr}$ in all subjects (sensitivity 0.800 , specificity 0.963 ).

Conclusions To the best of our knowledge, this is the first report incicating that the measurement of U-LFABP can be beneficial for in the diagnosis of ACS.
\end{abstract}

Key words: urinary liver fatty acid-binding protein, stable angina, acute coronary syndrome, percutaneous coronary intervention

(Inter Med 48: 1731-1737, 2009)

(DOI: 10.2169/internalmedicine.48.2410)

\section{Introduction}

Fatty acid-binding proteins (FABP) have recently been recognized as promising biomarkers. These small cytoplasmic proteins are abundantly expressed in tissues with active fatty acid metabolism. There are nine distinct types of FABP with a tissue-specific distribution, including the liver, intestine, muscle, heart, adipocyte, epidermis, ileum, brain, myelin and testis (1). Heart-type (H)-FABP is a diagnostic and prognostic marker for acute and chronic cardiac injury (2). In the kidney, epithelial cells of the proximal tubules express mRNA of liver-type (L)-FABP, and a certain amount is excreted into urine. Tubulo-interstitial inflammation induced by lipid toxicity may be provoked by stresses such as ischemia and toxins (3). Urinary (U)-L-FABP has been shown to be an excellent clinical marker that can be used to help predict and monitor the progression of renal disease (4).

Mild to moderate renal impairment has also been shown 
to be strongly associated with cardiovascular morbidity and mortality in patients with atherosclerotic disease and in the general population (5). Cardiovascular disease (CVD) and renal dysfunction frequently coexist, and this is referred to as the cardio-renal syndrome. This syndrome represents the sum total of an adversarial relation and is clinically manifest as worsening renal function. Despite the growing recognition of the frequent presentation of this syndrome, its underlying pathophysiology is not well understood. U-L-FABP might also be associated with cardio-renal syndrome. In fact, a higher level of U-L-FABP is considered to be an independent risk factor for the progression of renal disease (4). On the other hand, it is not known whether U-L-FABP reflects cardiovascular injury or whether cardiovascular injury increases the excretion of U-L-FABP.

Therefore, the aims of the present study were to evaluate the diagnostic value of U-L-FABP in patients with acute coronary syndrome (ACS) or stable angina pectoris (SAP) and to compare it with that of the estimated glomerular filtration rate (eGFR) and other biochemical indicators.

\section{Methods}

\section{Study population}

A total of 27 patients (19 men and 8 women, mean age $70 \pm 11$ years) with SAP ( $n=15$, SAP group) or ACS $(n=12$, ACS group) who had coronary angiograms and significant coronary stenosis ( $>50 \%$ luminal narrowing) as defined by coronary angiography and who were successfully implanted with a stent from November 2006 to October 2007 were enrolled in this study. Exclusion criteria included cardiac shock, age less than 30, pregnancy, comorbidity, prior percutaneous coronary intervention (PCI) within the previous 6 months or coronary artery bypass grafting within 2 months, current or future need for warfarin, need for chronic daily nonsteroidal anti-inflammatory drugs, creatinine $(\mathrm{Cr})$ more than $1.6 \mathrm{mg} / \mathrm{dL}$, and a calculated $\mathrm{Cr}$ clearance of less than $40 \mathrm{~mL} / \mathrm{min}$. ACS patients had ischemic discomfort at rest that lasted 5 minutes with an onset within 72 hours of randomization and associated with 1 of the following features: new or presumably new ST segment deviation of $0.5 \mathrm{~mm}$, T-wave inversion of $3 \mathrm{~mm}$ in 3 leads or left bundle branch block, and positive cardiac markers. SAP patients presented with angina pectoris, or had a positive stress test and had clinically significant angiographic stenosis in a native coronary vessel with a percent diameter stenosis of more than $50 \%$. In addition, 12 age-, sex-, body mass index (BMI)and several coronary risk factors-matched patients without CVD were also enrolled as controls (C group). The ethics committee of Fukuoka University Hospital approved this study and written informed consent was obtained from each patient.

Patients did not have vascular disease (aortitis treated by prednisolone) or hepatic dysfunction (viral and nonviral, transaminases more than three times the normal value). $\mathrm{Pa}$ - tients with low-density lipoprotein cholesterol (LDL-C) $\geq$ $140 \mathrm{mg} / \mathrm{dL}$ or triglyceride (TG) $\geq 150 \mathrm{mg} / \mathrm{dL}$ were diagnosed as dyslipidemia. Patients with systolic or diastolic blood pressure (SBP or DBP) $\geq 140 \mathrm{mmHg}$ or $90 \mathrm{mmHg}$ or who were under antihypertensive treatment were considered to have hypertension (HT). Patients who were being treated for diabetes mellitus (DM) or who had symptoms of DM and a fasting blood glucose concentration $\geq 126 \mathrm{mg} / \mathrm{dL}$ were considered to have DM. Otherwise, the results of a 75$\mathrm{g}$ oral glucose tolerance test were used to diagnose DM.

\section{Urine and blood sampling}

The first urine immediately before PCI, the second urine immediately after PCI and the third urine the morning after PCI were collected and centrifuged at $4{ }^{\circ} \mathrm{C}$ to remove sediments. The supernatant was stored at $-20^{\circ} \mathrm{C}$ until the assays. The concentration of U-L-FABP was determined in duplicate by specific enzyme immunoassays (CMIC, Tokyo, Japan) according to the manufacturer's instructions. At our laboratory, the intra- and inter-assay coefficients of variation were each $5 \%$, and the value was expressed as a ratio of the concentration of U-L-FABP to U-Cr (U-L-FABP/U-Cr) as measured by colorimetry. Urinary concentrations of albumin (U-Alb) were measured by nephelometry and latex photometric immunoassay, and the values were also related to the U-Cr concentration. Venous blood samples were obtained immediately before and after PCI and the morning after PCI.

Blood was collected in tubes with or without ethylenediaminetetraacetic acid (EDTA). Plasma was separated by centrifugation at $4{ }^{\circ} \mathrm{C}$ and stored at $-80^{\circ} \mathrm{C}$ until assays. Serum (S) Cr, lipid profile, uric acid (UA), high-sensitive Creactive protein (CRP), free fatty acid (FFA) were analyzed. When CRP was less than $0.3 \mathrm{mg} / \mathrm{dL}$, hs-CRP was performed. Estimated glomerular filtration rate (eGFR) was calculated according to the Modification of Diet in Renal Disease (6). Plasma levels of BNP and hemoglobin A1c (HbA1c) were also measured.

\section{PCI procedures and antiplatelet medications}

The intervention procedure was performed according to the standard technique by several operators. The endpoint of the procedure for the main vessel was TIMI 3, angiographic percentage of diameter stenosis $<10$ and no major dissection that would compromise flow in the vessel. During the procedure, patients received intravenous heparin to maintain an activated clotting time of more than 250 seconds. Most patients were pretreated with ticlopidine or clopidogrel and aspirin at least 48 hours before PCI. A loading dose of 300 $\mathrm{mg}$ of clopidogrel was given to 4 ACS patients who had not previously been taking antiplatelet agents. After the procedure, aspirin (100 mg/day) and clopidogrel $(75 \mathrm{mg} /$ day $)$ were continued. The patients in the $\mathrm{C}$ group did not have antiplatelet medications. 
Table 1. Baseline Patient Characteristics

\begin{tabular}{|c|c|c|c|c|c|c|}
\hline & \multirow{2}{*}{$\begin{array}{l}\text { ACS } \\
\text { group }\end{array}$} & \multirow{2}{*}{$\begin{array}{l}\text { SAP } \\
\text { group }\end{array}$} & \multirow{2}{*}{ C group } & \multicolumn{3}{|c|}{$p$ value } \\
\hline & & & & ACS vs. SAP & ACS vs. C & SAP vs. C \\
\hline Age(year) & $72 \pm 12$ & $69 \pm 12$ & $65 \pm 8$ & 0.752 & 0.309 & 0.441 \\
\hline BMI & $23.0 \pm 4.6$ & $24.3 \pm 2.5$ & $23.8 \pm 1.5$ & 0.515 & 0.746 & 0.678 \\
\hline Male(\%) & 75 & 67 & 67 & 0.653 & 0.560 & 0.312 \\
\hline $\mathrm{SBP}(\mathrm{mmHg})$ & $132 \pm 31$ & $129 \pm 19$ & $123 \pm 15$ & 0.791 & 0.401 & 0.376 \\
\hline $\mathrm{DBP}(\mathrm{mmHg})$ & $74 \pm 10$ & $74 \pm 12$ & $75 \pm 15$ & 0.977 & 0.833 & 0.802 \\
\hline $\mathrm{EF}(\%)$ & $58 \pm 12$ & $63 \pm 16$ & & 0.462 & & \\
\hline Family history(\%) & 8 & 0 & 0 & 0.255 & & \\
\hline Diabetes mellitus(\%) & 47 & 40 & 67 & 0.930 & & \\
\hline Dyslipidemia(\%) & 58 & 80 & 75 & 0.221 & & \\
\hline Hypertension(\%) & 58 & 87 & 75 & 0.930 & & \\
\hline Current smoker $(\%)$ & 50 & 33 & 25 & 0.381 & & \\
\hline Hyper uricemia(\%) & 42 & 13 & 17 & 0.095 & & \\
\hline contrast medium dose $(\mathrm{mL})$ & $174 \pm 44$ & $155 \pm 47$ & & 0.226 & & \\
\hline Pre TIMI & $47 \pm 8$ & $23 \pm 9$ & & 0.002 & & \\
\hline Post TIMI & $15 \pm 6$ & $20 \pm 7$ & & 0.457 & & \\
\hline TypeB2/C(\%) & 67 & 87 & & 0.214 & & \\
\hline Lesion (vessel) & $1.7 \pm 0.8$ & $1.7 \pm 0.8$ & & & & \\
\hline \multicolumn{7}{|l|}{ Culplit } \\
\hline $\mathrm{RCA}(\%)$ & 8 & 27 & & & & \\
\hline $\operatorname{LAD}(\%)$ & 67 & 53 & & & & \\
\hline $\operatorname{LCX}(\%)$ & 25 & 20 & & & & \\
\hline \multicolumn{7}{|l|}{ Medication } \\
\hline Statin (\%) & 50 & 80 & 58 & 0.100 & & \\
\hline ARB $(\%)$ & 67 & 80 & 58 & 0.432 & & \\
\hline CCB $(\%)$ & 17 & 73 & 58 & 0.003 & & \\
\hline ACEI (\%) & 0 & 7 & 33 & 0.362 & & \\
\hline$\beta$ bocker (\%) & 17 & 13 & 8 & 0.809 & & \\
\hline Diuretics (\%) & 17 & 20 & 8 & 0.825 & & \\
\hline Nitrol (\%) & 8 & 20 & 0 & 0.397 & & \\
\hline Nicorandil (\%) & 17 & 40 & 0 & 0.187 & & \\
\hline
\end{tabular}

BMI, body mass index; SBP, systolic blood pressure; DBP, diastolic blood pressure; EF, ejection fraction; RCA, right coronary artery; LAD, left anterior descending artery; LCX, left circumflex artery; ARB, angiotensin II receptor blockers; $\mathrm{CCB}$, calcium channel blocker; ACEI, angiotensin converting enzyme inhibitor

\section{Thrombolysis in myocardial infarction (TIMI) frame}

The angiographic core laboratory at Fukuoka University Hospital analyzed all pre- and post-procedural findings using a CMS-GFT system (MEDIS, The Netherlands). All measurements were performed on angiographic images recorded after the intra-coronary administration of nitroglycerin.

\section{Statistical analysis}

Statistical analysis was performed using the Excel 2003 (SSRI, Tokyo, Japan) and Stat View statistical software package (Stat View 5; SAS Institute Inc., Cary, NC, USA). Categorical and continuous variables were compared by a chi-square analysis and one-way analysis of variance followed by a post-hoc Fisher's PLSD test. A multiple regression analysis was performed to analyze the relationship between the classification of SAP and ACS and significant factors. Receiver-operating characteristic (ROC) curve analysis was used to determine the cut-off of the U-L-FABP to distinguish between SAP + controls and ACS at the highest possible sensitivity and specificity levels. Data are presented as the mean and standard deviation (SD). Significance was considered to be a $\mathrm{p}$ value of less than 0.05 unless indicated otherwise.

\section{Results}

\section{Patient characteristics}

Baseline patient characteristics are shown in Table 1. There were no significant differences in age, the percentage of males, or the number of coronary risk factors such as DM, HT, and smoking among the ACS, SAP and C groups. In addition, the contrast media dose in the SAP group was similar to that in the ACS group. There was a significant difference in TIMI frame between the ACS and SAP groups $(\mathrm{p}=0.0012)$. In addition, the ACS group received more CCB than the SAP group $(\mathrm{p}=0.003)$.

\section{Changes in parameters in urine}

U-L-FABP/U-Cr and U-NAG before and after PCI and 1 day after PCI are shown in Fig. 1a and 1b. U-L-FABP/U-Cr in the ACS group $(61.0 \pm 67.1 \mu \mathrm{g} / \mathrm{g} \cdot \mathrm{Cr})$ was significantly 

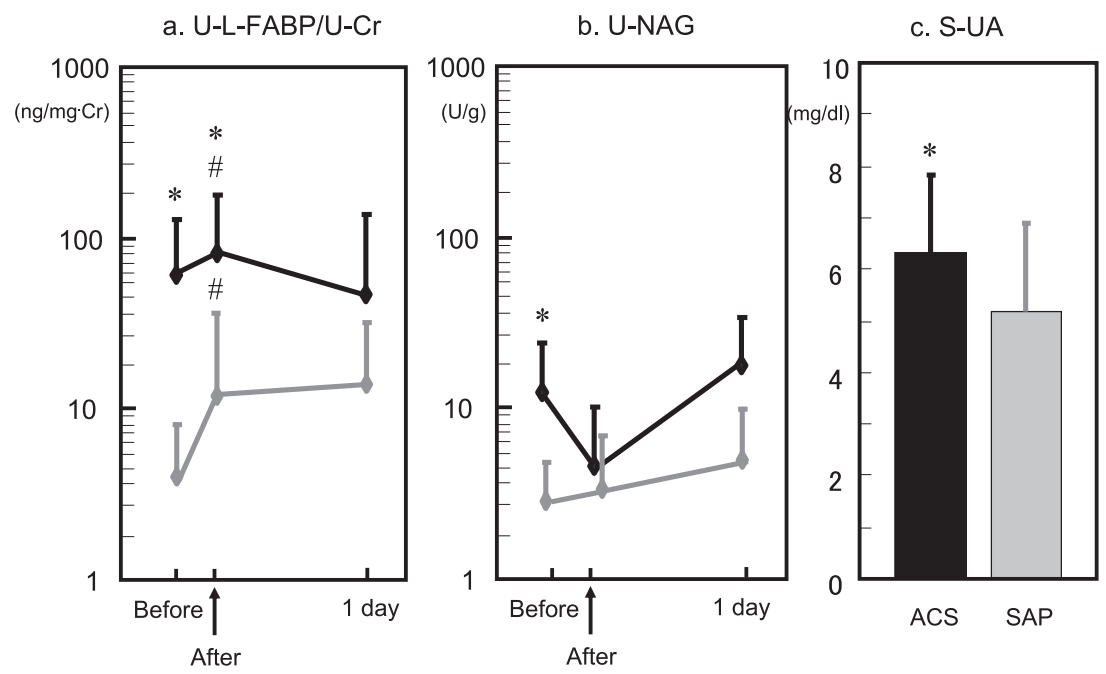

Figure 1. Changes in a) U-L-FABP/U-Cr, b) U-NAG in the ACS (black line) and SAP (gray line) groups. c) Levels of S-UA before PCI in the ACS and SAP groups. "p<0.05 vs. SAP group. ${ }^{\#} \mathbf{p}<0.05$ vs. before PCI.

higher than those in the SAP $(3.8 \pm 3.8 \mu \mathrm{g} / \mathrm{g} \cdot \mathrm{Cr}, \mathrm{p}=0.003)$ and C groups $(6.5 \pm 6.4 \mu \mathrm{g} / \mathrm{g} \cdot \mathrm{Cr}, \mathrm{p}=0.001)$ before PCI, and the levels in the ACS group was after PCI. Although ACS group received more $\mathrm{CCB}$ than the SAP group, there were no changes in U-L-FABP/U-Cr throughout the study period between CCB and non-CCB therapies (data not shown). In addition, although there was no difference in U-NAG between the SAP and $\mathrm{C}$ groups $(2.7 \pm 2.1$ vs. $3.2 \pm 2.4 \mathrm{U} / \mathrm{g}$, respectively) before PCI, U-NAG before PCI in the ACS group $(12.3 \pm 11.6 \mathrm{U} / \mathrm{g})$ was significantly higher than that in the SAP group ( $\mathrm{p}=0.037)$. On the other hand, there was no significant difference in the ratio of U-Alb to U-Cr (U-Alb/U$\mathrm{Cr})$ between the SAP $(6.9 \pm 5.6 \mathrm{mg} / \mathrm{g} \cdot \mathrm{Cr})$ and ACS $(8.2 \pm 7.2$ $\mathrm{mg} / \mathrm{g} \cdot \mathrm{Cr})$ groups $(\mathrm{p}=0.679)$. U-Alb before PCI could not be measured in 6 of 13 patients $(42 \%)$ in the ACS group and in 2 of 15 patients $(13 \%)$ in the SAP group because it was outside the range of measurement $(<30 \mathrm{~g} / \mathrm{dL}$ or $>300 \mathrm{~g} / \mathrm{dL})$ in our assay system.

\section{Changes in parameters in blood}

Serum uric acid (S-UA) in the ACS group was significantly greater than that in the SAP group $(\mathrm{p}=0.046)$ (Fig. 1c), while there were no significant differences in lipid profile, eGFR, BNP or troponin $\mathrm{T}$ between the ACS and SAP groups (Table 2). In addition, FFA levels in the ACS and SAP groups were significantly higher than that in the $C$ group ( $\mathrm{p}=0.003$ ), and BNP in the ACS group was significantly greater than that in the $\mathrm{C}$ group $(\mathrm{p}=0.012)$.

\section{Association between the classification of SAP and $A C S$ and significant factors immediately before $P C I$}

Since U-L-FABP/U-Cr, U-NAG and S-UA before PCI in the ACS group were significantly higher than those in the SAP group, and since \% medication with CCB in the ACS group was significantly lower than that in the SAP group, a multiple regression analysis was performed to analyze the relationship between the classification of SAP and ACS and these significant factors (Table 3). U-L-FABP/U-Cr was most closely associated with the classification of SAP and ACS $(\mathrm{p}<0.0001)$.

\section{Sensitivity and specificity of the level of U-L-FABP/U- Cr for the diagnosis of ACS}

For all subjects, the sensitivity and specificity of U-LFABP were examined by an ROC analysis, as shown in Fig. 2. The cut-off level of U-L-FABP that gave the greatest sensitivity and specificity for the diagnosis of ACS was 13.4 $\mu \mathrm{g} / \mathrm{g} \cdot \mathrm{Cr}$ (sensitivity 0.800 , specificity 0.963 ).

\section{Discussion}

In this study, we assessed the diagnostic value of U-LFABP in patients with ACS and compared it with that of eGFR and other biochemical parameters. The major findings of the present study are as follows: 1) U-L-FABP/U-Cr in the ACS group was significantly higher than that in the SAP group immediately before and after PCI, 2) regardless of the increase in U-L-FABP/U-Cr in the ACS group, there were no cases of contrast-induced nephropathy (CIN) according to the value of S-Cr, 3) U-L-FABP/U-Cr was most closely associated with the classification of SAP and ACS using a multiple regression analysis, and 4) when the cut-off level of U-L-FABP/U-Cr for the diagnosis of ACS was $13.4 \mu \mathrm{g} / \mathrm{g} \cdot \mathrm{Cr}$ in all subjects (sensitivity 0.800 , specificity 0.963 ).

We determined the factors that were related to the classification of SAP and ACS because an increase in U-L-FABP/ $\mathrm{U}-\mathrm{Cr}$ was seen in patients with ACS. The results of a logistic regression analysis showed that the classification of SAP and ACS most strongly contributed to U-L-FABP/U-Cr. Although the 24-hour urinary excretion of Alb was significantly higher in acute myocardial infarction (AMI) than in the control group after adjustment (7), we could not confirm 
Table 2. Parameters in Blood

\begin{tabular}{|c|c|c|c|c|c|c|}
\hline & \multirow{2}{*}{$\begin{array}{l}\text { ACS } \\
\text { group }\end{array}$} & \multirow{2}{*}{$\begin{array}{l}\text { SAP } \\
\text { group }\end{array}$} & \multirow[t]{2}{*}{$C$ group } & \multicolumn{3}{|c|}{$p$ value } \\
\hline & & & & ACS vs. SAP & ACS vs. C & SAP vs $C$ \\
\hline $\mathrm{CRP}(\mathrm{mg} / \mathrm{dL})$ & $0.5 \pm 0.8$ & $0.45 \pm 1.1$ & $0.17 \pm 0.26$ & 0.891 & 0.1731 & 0.402 \\
\hline Insulin $(\mu \mathrm{U} / \mathrm{mL})$ & $10.2 \pm 4.6$ & $12.9 \pm 16.5$ & $14.4 \pm 10.3$ & 0.592 & 0.138 & 0.675 \\
\hline Glu (mg/dL) & $127 \pm 32$ & $137 \pm 63$ & $141 \pm 36$ & 0.603 & 0.282 & 0.805 \\
\hline $\mathrm{TG}(\mathrm{mg} / \mathrm{dL})$ & $116 \pm 66$ & $125 \pm 55$ & $118 \pm 45$ & 0.707 & 0.937 & 0.726 \\
\hline LDL-C (mg/dL) & $110 \pm 23$ & $96 \pm 15$ & $100 \pm 32$ & 0.055 & 0.392 & 0.615 \\
\hline $\mathrm{HDL}-\mathrm{C}(\mathrm{mg} / \mathrm{dL})$ & $44 \pm 12$ & $40 \pm 10$ & $50 \pm 12$ & 0.402 & 0.22 & 0.391 \\
\hline UN (mg/dL) & $16 \pm 7$ & $15 \pm 7$ & $14 \pm 6$ & 0.807 & 0.476 & 0.526 \\
\hline $\mathrm{Cr}(\mathrm{mg} / \mathrm{dL})$ & $0.9 \pm 0.2$ & $0.8 \pm 0.2$ & $0.9 \pm 0.2$ & 0.537 & 0.752 & 0.82 \\
\hline $\operatorname{eGFR}\left(\mathrm{mL} / \mathrm{min} / 1.73 \mathrm{~m}^{2}\right)$ & $70.7 \pm 19.7$ & $74.7 \pm 19.7$ & $75.9 \pm 22.3$ & 0.507 & 0.559 & 0.88 \\
\hline $\mathrm{HbA} 1 \mathrm{c}(\%)$ & $6.4 \pm 1.5$ & $5.9 \pm 1.5$ & $5.9 \pm 0.7$ & 0.218 & 0.276 & 0.202 \\
\hline $\mathrm{FFA}(\mu \mathrm{Eq} / \mathrm{L})$ & $1246.5 \pm 779.7$ & $996.1 \pm 507.9$ & $511.4 \pm 332.1$ & 0.26 & 0.003 & 0.008 \\
\hline BNP $(p g / d L)$ & $122 \pm 133$ & $55 \pm 68$ & $29 \pm 22$ & 0.052 & 0.012 & 0.26 \\
\hline toroponinT $(\mathrm{ng} / \mathrm{mL})$ & $0.8 \pm 1.95$ & $0.018 \pm 0.04$ & $0.008 \pm 0.02$ & 0.07 & 0.08 & 0.4 \\
\hline $\mathrm{Cr}$ at dischrage $(\mathrm{mg} / \mathrm{dL})$ & $0.93 \pm 0.20$ & $0.89 \pm 0.23$ & & 0.471 & & \\
\hline CK (IU/L) & $223 \pm 320$ & $123 \pm 92$ & & 0.26 & & \\
\hline
\end{tabular}

CRP, C-reactive protein; Glu, glucose; TG, triglyceride; LDL-C, low-density lipoprotein cholesterol; HDL-C, high-density lipoprotein cholesterol; UN, urea nitrogen; $\mathrm{Cr}$, creatinine; eGFR, estimated glomerular filtration rate; HbA1c, hemoglobin A1c; FFA, free fatty acid; BNP, brain natriuretic peptide; CK, creatine kinase.

Table 3. Multiple Regression Analysis for Predicting Classification of SAP and ACS

\begin{tabular}{lcc}
\hline Factors & $\begin{array}{c}\text { Standardized } \\
\text { regression } \\
\text { coefficient }\end{array}$ & $\mathrm{p} \mathrm{value}$ \\
\hline U-L-FABP/U-Cr & -0.572 & $<0.0001$ \\
S-UA & -0.235 & 0.075 \\
U-NAG & -0.241 & 0.064 \\
Medication of CCB & 0.452 & 0.002 \\
\hline
\end{tabular}

U-L-FABP, urinary liver-fatty acid-binding protein; U$\mathrm{Cr}$, U-creatinine; S-UA, serum uric acid; U-NAG, U$\mathrm{N}$-acetyl- $\beta$-D-glucosaminidase; $\quad \mathrm{CCB}, \quad$ calcium channel blocker.

that there was a significant difference in U-Alb/U-Cr between the ACS and SAP groups in the present study because $42 \%$ of patients in the ACS group and $13 \%$ of patients in the SAP group showed U-Alb values outside the range of measurement. Thus, in our department, U-L-FABP/ $\mathrm{U}-\mathrm{Cr}$ may be a more useful parameter than $\mathrm{U}-\mathrm{Alb} / \mathrm{U}-\mathrm{Cr}$ for the classification of SAP and ACS.

U-L-FABP is upregulated by stresses such as ischemia, toxins and an increase in free fatty acids (FFAs) $(3,8)$. It is widely known that FFAs are increased in AMI $(9,10)$. Although ACS may induce stresses with an increase in FFAs and subsequently increase the excretion of U-L-FABP, there were no differences in FFAs between the ACS and SAP groups in the present study. Most serum FFAs are bound to Alb, and only a small amount of FFAs are unbound (FFAu). Serum FFAu was measured in 22 patients with PCI 5 minutes before and 30 minutes after the procedure and the latter value was 14 -fold higher than both the former value and

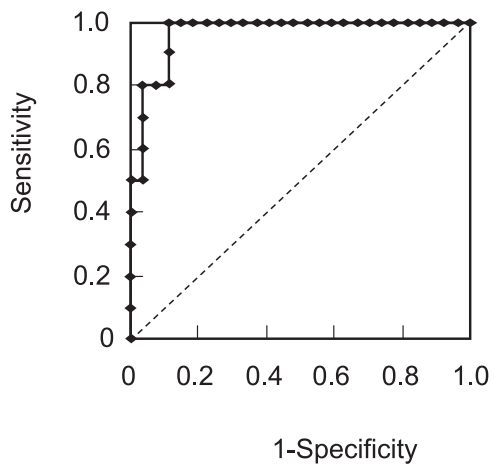

Figure 2. Receiver-operating characteristic (ROC) curve for U-L-FABP for the diagnosis of ACS in all subjects.

that in healthy volunteers (11). FFAu can be measured with recombinant FABP labeled with a fluorescent tag (12). Serum FFAu levels were measured in patients undergoing PCI, and the results indicated that FFAu levels may provide a more sensitive measure of angioplasty-induced ischemia (13). In addition, FFA concentrations, rather than total FFAu, do not provide a sensitive guide to the pathophysiology of underlying CVD. Since FFAu was a useful marker for initially identifying unstable angina patients $(14,15)$, it should be measured.

No clear evidence has been reported that oxidative stress and inflammation directly promote U-L-FABP excretion or increase FFAu, based on the results in coronary injury and ischemia. The increased vascular production of reactive oxygen species is a characteristic feature of vascular disease states, including CVD. Such overproduction may play an important role in the pathogenesis of ACS and subsequently affect the kidney and increase U-L-FABP levels. Therefore, U-L-FABP/U-Cr might be a good biomarker for acute cardio-renal syndrome under ACS because it showed a 
higher sensitivity and specificity for the diagnosis of ACS in this study.

The S-Cr level was measured from blood samples drawn at the time of PCI (baseline), 1 and 2 to 5 days after the procedure, and before discharge. $\mathrm{Cr}$ clearance $(\mathrm{CCr})$ was calculated by applying the Cockcroft-Gault formula, while adjusting for female sex. CIN is defined as an absolute increase in the S-Cr level of at least $0.5 \mathrm{mg} / \mathrm{dL} 2$ to 5 days after the procedure or a relative increase of at least $25 \%$ from the baseline value. Nakamura et al reported a high U-LFABP level in the CIN group (16). In this study, no patient in the ACS group showed CIN regardless of a high U-LFABP/U-Cr level. Although U-L-FABP levels after angiography usually showed either little change or an increase, UL-FABP/U-Cr decreased in the ACS group 1 day after PCI. This indicates that the increase in U-L-FABP between before and after PCI did not result from CIN. This may be due to the onset of ACS. U-L-FABP/U-Cr is also a useful biomarker for predicting the occurrence of CIN in patients with SAP, but not ACS.

The level of U-L-FABP can be affected by treatment with various compounds, such as ARBs (17), calcium channel blocker (CCB) (18), statin (19) and peroxisome proliferatoractivated receptor (PPAR)- $\gamma$ agonist (20). CCB, azelnidipine and statin reduced oxidative stress and L-FABP (20-33\% after six months), while amlodipine did not reduce L-FABP $(18,19)$. In this study, there was no significant difference in the percentage of patients treated with statin or ARB between the ACS and SAP groups, and none of the patients received PPAR- $\gamma$ agonist. Although the ACS group received more CCB than the SAP group $(\mathrm{p}=0.003)$, there were no changes in U-L-FABP/U-Cr throughout the study period between $\mathrm{CCB}$ and non-CCB therapies and none of the patients were treated with azelnidipine.

Two patients in the ACS group initially received diuretics due to congestive heart failure (CHF). BNP levels in the ACS group were higher than those in the SAP group, but the difference was not significant. We do not have data on L-FABP in patients with $\mathrm{CHF}$, and further analysis is needed to confirm whether L-FABP is increased in CHF.

\section{Study limitations}

This study has three important limitations. First, the sample size was relatively small, which limited our ability to determine significance. A large prospective trial is warranted to evaluate the potential benefits of U-L-FABP in CVD. Second, Kato et al reported that cystatin C, which is a cysteine protease inhibitor, was useful for predicting the occurrence of CIN (21). In addition, Sasaki et al (22) reported that U-L-FABP levels may reflect the stress induced by FFA on the proximal tubules, which could lead to severe tubulointerstitial damage. Further analysis is needed to confirm whether cystatin $\mathrm{C}$ or U-FFA is increased. Finally, the U-LFABP level was not analyzed from 2 to 5 days after PCI in this study. Despite these limitations, our data suggest that the measurement of U-L-FABP may aid in the diagnosis of ACS.

In conclusion, to the best of our knowledge, this is the first report indicating that the measurement of U-L-FABP may aid in the diagnosis of ACS. It may also be a useful biomarker for predicting the occurrence of CIN in patients with SAP, but not ACS.

\section{References}

1. Glatz JF, van der Vusse GJ. Cellular fatty acid-binding proteins: their function and physiological significance. Prog Lipid Res 35: 243-282, 1996.

2. Glatz JF, van Bilsen M, Paulussen RJ, et al. Release of fatty acidbinding protein from isolated rat heart subjected to ischemia and reperfusion or to the calcium paradox. Biochim Biophys Acta 961: 148-152, 1988.

3. Ramsammy LS, Josepovitz C, Lane BP, et al. Polyaspartic acid protects against gentamicin nephrotoxicity in the rat. J Pharmacol Exp Ther 250: 149-153, 1989.

4. Kamijo A, Sugaya T, Hikawa A, et al. Urinary excretion of fatty acid-binding protein reflects stress overload on the proximal tubules. Am J Pathol 165: 1243-1255, 2004.

5. Mann JF, Gerstein HC, Pogue J, et al. Renal insufficiency as a predictor of cardiovascular outcomes and the impact of ramipril: the HOPE randomized trial. Ann Intern Med 134: 629-636, 2001.

6. Matsuo S, Imai E, Horio M, et al. Collaborators developing the Japanese equation for estimated GFR. Revised equations for estimated GFR from serum creatinine in Japan. Am J Kidney Dis 53: 982-992, 2009.

7. Berton G, Citro T, Palmieri R, et al. Albumin excretion rate increases during acute myocardial infarction and strongly predicts early mortality. Circulation 96: 3338-3345, 1997.

8. Matthys E, Patel Y, Kreisberg J, et al. Lipid alterations induced by renal ischemia: pathogenic factor in membrane damage. Kidney Int 26: 153-161, 1984.
9. Oliver MF, Opie LH. Effects of glucose and fatty acids on myocardial ischaemia and arrhythmias. Lancet 343: 155-158, 1994.

10. Kurien VA, Oliver MF. Serum-free-fatty-acids after acute myocardial infarction and cerebral vascular occlusion. Lancet 2: 122-127, 1966.

11. Cantor WJ, Kim HH, Jolly S, et al. B-type natriuretic peptide and serum unbound free fatty acid levels after contemporary percutaneous coronary intervention. J Invasive Cardiol 20: 186-188, 2008.

12. Richieri GV, Ogata RT, Kleinfeld AM. A fluorescently labeled intestinal fatty acid binding protein. Interactions with fatty acids and its use in monitoring free fatty acids. J Biol Chem 267: 2349523501, 1992.

13. Brezis M, Rosen S. Hypoxia of the renal medulla--its implications for disease. N Engl J Med 332: 647-655, 1995.

14. Kleinfeld AM, Prothro D, Brown DL, et al. Increases in serum unbound free fatty acid levels following coronary angioplasty. Am J Cardiol 78: 1350-1354, 1996.

15. Azzazy HM, Pelsers MM, Christenson RH. Unbound free fatty acids and heart-type fatty acid-binding protein: diagnostic assays and clinical applications. Clin Chem 52: 19-29, 2006.

16. Nakamura T, Sugaya T, Node K, et al. Urinary excretion of livertype fatty acid-binding protein in contrast medium-induced nephropathy. Am J Kidney Dis 47: 439-444, 2006.

17. Nakamura T, Sugaya T, Kawagoe $Y$, et al. Candesartan reduces urinary fatty acid-binding protein excretion in patients with 
autosomal dominant polycystic kidney disease. Am J Med Sci 330: 161-165, 2005.

18. Nakamura T, Sugaya $T$, Kawagoe $Y$, et al. Azelnidipine reduces urinary protein excretion and urinary liver-type fatty acid binding protein in patients with hypertensive chronic kidney disease. Am J Med Sci 333: 321-326, 2007.

19. Nakamura T, Sugaya T, Kawagoe $Y$, et al. Effect of pitavastatin on urinary liver-type fatty acid-binding protein levels in patients with early diabetic nephropathy. Diabetes Care 28: 2728-2732, 2005.

20. Nakamura T, Sugaya $T$, Kawagoe $Y$, et al. Effect of pioglitazone on urinary liver-type fatty acid-binding protein concentrations in diabetes patients with microalbuminuria. Diabetes Metab Res Rev 22: 385-389, 2006

21. Kato K, Sato N, Yamamoto T, Iwasaki YK, Tanaka K, Mizuno K. Valuable markers for contrast-induced nephropathy in patients undergoing cardiac catheterization. Circ J 72: 1499-1505, 2008.

22. Sasaki H, Kamijo-Ikemori A, Sugaya T, et al. Urinary fatty acids and liver-type fatty acid binding protein in diabetic nephropathy. Nephron Clin Pract 112: c148-c156, 2009.

(C) 2009 The Japanese Society of Internal Medicine http://www.naika.or.jp/imindex.html 